\title{
SYSTEMS WITH MONOTONE AND SLOPE RESTRICTED NONLINEARITIES
}

\author{
VLADIMIR RĂSVAN
}

\begin{abstract}
The paper starts from the suggestion of R. E. Kalman that additional information on nonlinearity slope may improve the sufficient conditions for absolute stability. This leads to the so called systems with augmented dynamics. Motivated also by the problem of the PIO II aircraft oscillations - self sustained oscillations induced by the saturation nonlinearities, which are both sector and slope restricted - the paper considers a generalization of the Yakubovich criterion to the case of the systems with critical and unstable linear part. The same generalization concerns a quite well known stability criterion where only slope restrictions are taken into account: the published version is improved by using all advantages of the Liapunov method and of the frequency domain stability inequalities. The results are illustrated by several applications.
\end{abstract}

\section{Physical and engineering motivation}

In a standard control system the basic structure is a feedback system, e.g., Fig. 1, where the linear subsystem denoted by $H_{a}(s)$ accounts for the controlled plant while the subsystem denoted by $H_{c}(s)$ accounts for the controller; $H_{a}(s)$ and $H_{c}(s)$ are the transfer functions of the subsystems; they are associated to the descriptions by forced (controlled) linear ordinary differential equations with constant coefficients in a standard way, e.g., [15] and we skip other explanation.

This feedback structure is rather general. In such an important application as the problem of $\mathrm{PIO}-\mathrm{P}$ (ilot) $\mathrm{I}$ (n-the-loop) $\mathrm{O}$ (scillations) the subsystem $H_{a}(s)$ accounts for airframe dynamics while $H_{c}(s)$ accounts for pilot dynamics (the dynamics of a human operator). We continue with this rather special application because it is extremely relevant for our way of problem development. The structure of Fig. 1 describes a linear system which corresponds to PIO I case

(c) 2011 Mathematical Institute, Slovak Academy of Sciences.

2010 Mathematics Subject Classification: 34D23, 93D10, 93D30.

Keywords: nonlinear systems, slope restrictions, augmented dynamics, frequency domain inequalities.

This work was supported by CNCSIS-UEFISCSU project number PN II - IDEI 95/2007. 
VLADIMIR RĂSVAN

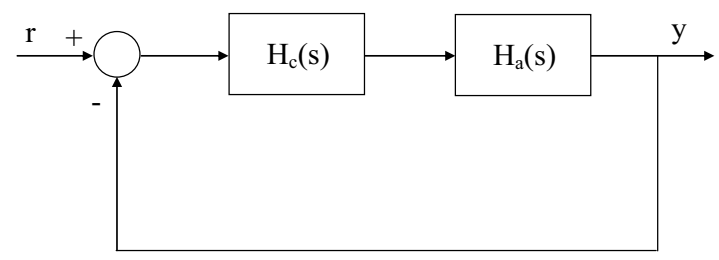

Figure 1. Basic linear feedback structure.

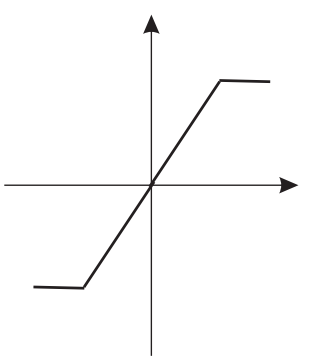

a)

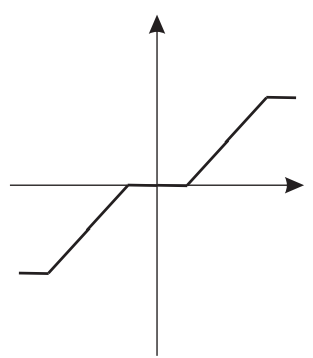

b)

FIGURE 2. Saturation nonlinearities: a) standard; b) with deadzone.

when the entire structure is linear and self sustained oscillations occur for a pair of purely imaginary eigenvalues of the overall system. There are however PIO cases when the frequency of the oscillations does not coincide with some possible purely imaginary eigenvalues of the linear system. Such real case data point to nonlinear phenomena and the simplest approach is to consider saturation of the actuators or, as it is better known by aircraft engineers and pilots, onset of position and rate limiters. The saturation functions are displayed in Fig. 2 while the structure with rate limiter is shown in Fig. 3. This is called PIO II [1].

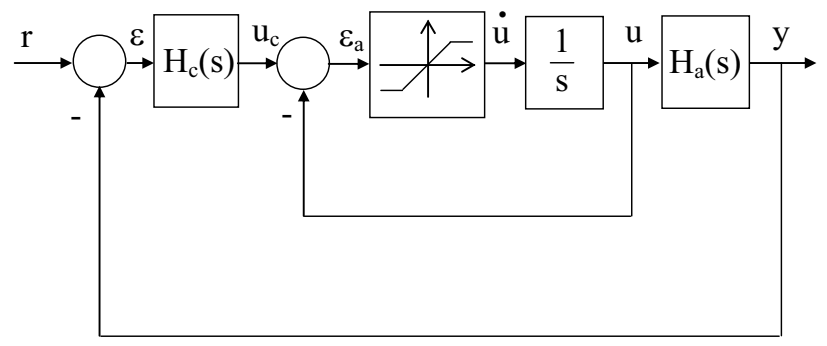

FiguRE 3. Feedback structure with rate limiter. 


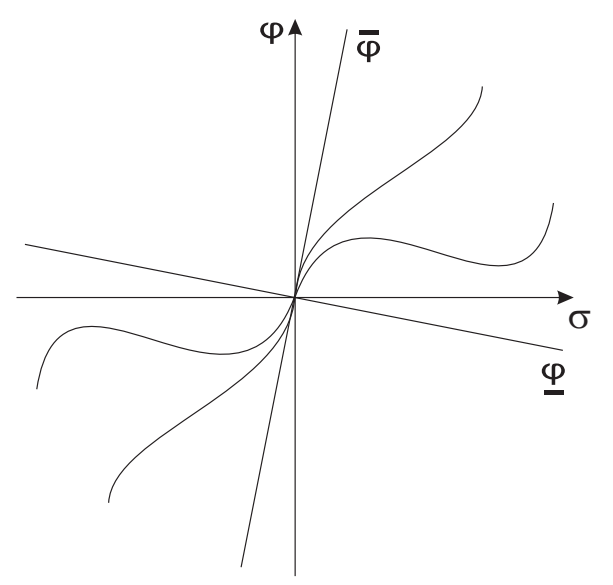

Figure 4. Sector restricted nonlinearity.

A simple examination of the graphics of Fig. 2 shows that these functions are sector restricted, i.e., belong to the class described by Fig. 4 .

\section{Problem statement. Approaches and extensions}

A. Following the line of the previous section we start from the structure of Fig. 3. Consider that the linear subsystems denoted by their transfer functions $H_{a}(\sigma)$ and $H_{c}(\sigma)$ are the standard ones in Control Theory, i.e., described by rational functions which are strictly proper for $H_{a}(\sigma)$ and at most proper for $H_{c}(\sigma)$ :

$$
H_{a}(\sigma)=\frac{N_{a}(\sigma)}{D_{a}(\sigma)}, \partial\left(N_{a}\right)<\partial\left(D_{a}\right) ; \quad H_{c}(\sigma)=\frac{N_{c}(\sigma)}{D_{c}(\sigma)}, \partial\left(N_{c}\right) \leq \partial\left(D_{c}\right),
$$

where $\partial(\cdot)$ denotes the polynomial degree. We consider the state representations of the two transfer functions

$$
\begin{aligned}
& \dot{x}_{a}=A_{a} x_{a}+b_{a} \mu_{a}(t), \quad \nu_{a}=c_{a}^{*} x_{a}, \\
& c_{a}\left(\sigma I-A_{a}\right)^{-1} b_{a} \equiv H_{a}(\sigma)=\frac{N_{a}(\sigma)}{D_{a}(\sigma)}
\end{aligned}
$$

and

$$
\begin{gathered}
\dot{x}_{c}=A_{c} x_{c}+b_{c} \mu_{c}(t), \quad \nu_{c}=c_{c}^{*} x_{c}+\gamma_{c} \mu_{c}, \\
c_{c}\left(\sigma I-A_{c}\right)^{-1} b_{c}+\gamma_{c} \equiv H_{c}(\sigma)=\frac{N_{c}(\sigma)}{D_{c}(\sigma)}
\end{gathered}
$$

connected as in Fig. 3 


$$
\dot{\mu}_{a}=f\left(\nu_{c}-\mu_{a}\right), \quad \mu_{c}=\nu_{0}-\nu_{a},
$$

where $\nu_{0}$ is the so called reference signal. Consequently, the system of Fig. 3 is described by

$$
\begin{aligned}
& \dot{x}_{a}=A_{a} x_{a}+b_{a} \mu_{a}, \\
& \dot{x}_{c}=-b_{c} c_{a}^{*} x_{a}+A_{c} x_{c}+b_{c} \nu_{0}, \\
& \dot{\mu}_{a}=f\left(\nu_{c}-\mu_{a}\right) ; \quad \nu_{c}=-\gamma_{c} c_{a}^{*} x_{a}+f_{c}^{*} x_{c}+\gamma_{c} \nu_{0} .
\end{aligned}
$$

Let $\nu_{0} \equiv$ const; if $f(\nu)=0$ for $\nu=0$ only, then (15) has a constant solution (equilibrium) which may be computed as solution of the following linear system

$$
\left(\begin{array}{ccc}
A_{a}-\gamma_{c} b_{a} c_{a}^{*} & b_{a} f_{c}^{*} & 0 \\
-b_{c} c_{a}^{*} & A_{c} & 0 \\
\gamma_{c} c_{a}^{*} & -f_{c}^{*} & 1
\end{array}\right)\left(\begin{array}{c}
\bar{x}_{a} \\
\bar{x}_{c} \\
\bar{\mu}_{a}
\end{array}\right)=\left(\begin{array}{c}
-\gamma_{c} b_{a} \\
b_{c} \\
\gamma_{c}
\end{array}\right) \nu_{0} .
$$

If the system in deviations is introduced, then the deviation variables

$$
z_{a}=x_{a}-\bar{x}_{a}, \quad z_{c}=x_{c}-\bar{x}_{c}, \quad \xi_{a}=\mu_{a}-\bar{\mu}_{a}
$$

satisfy

$$
\begin{aligned}
& \dot{z}_{a}=A_{a} z_{a}+b_{a} \xi_{a}, \\
& \dot{z}_{c}=-b_{c} c_{a}^{*} z_{a}+A_{c} z_{c}, \\
& \dot{\xi}_{a}=f\left(-\gamma_{c} c_{a}^{*} z_{a}+f_{c}^{*} z_{c}-\xi_{a}\right) .
\end{aligned}
$$

Denoting $\phi(\lambda)=-f(-\lambda)$ we obtain

$$
\begin{aligned}
& \dot{z}_{a}=A_{a} z_{a}+b_{a} \xi_{a}, \\
& \dot{z}_{c}=-b_{c} c_{a}^{*} z_{a}+A_{c} z_{c}, \\
& \dot{\xi}_{a}=-\phi\left(\gamma_{c} c_{a}^{*} z_{a}-f_{c}^{*} z_{c}+\xi_{a}\right) .
\end{aligned}
$$

Obviously, this system belongs to the class described by

$$
\dot{x}=A x-b \phi\left(c^{*} x\right)
$$

with suitable notations. By writing

$$
\begin{aligned}
& \dot{x}=A x+b \mu(t), \quad \nu=c^{*} x, \\
& \mu=-\phi(\nu)
\end{aligned}
$$

we obtain a systemic form for (10) which corresponds to the general structure of Fig. 罒

The nonlinear function $\phi(\cdot)$ is assumed to be as in Fig. 4 hence it is subject to the basic sector restrictions

$$
\underline{\varphi} \leq \frac{\phi(\nu)}{\nu} \leq \bar{\varphi} .
$$

We are now in position to state, for the sake of completeness. 


\section{SLOPE RESTRICTED NONLINEARITIES}

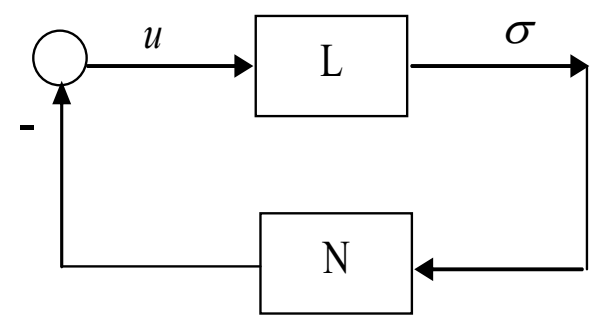

FiguRE 5. Absolute stability feedback structure.

Problem (Absolute stability). Given the system (10), find conditions on $(A, b, c$, $\underline{\varphi}, \bar{\varphi})$ in order that the zero equilibrium of the system, i.e., the identically zero solution, should be globally asymptotically stable for all nonlinear functions satisfying (12).

It is worth mentioning, once more, that the absolute stability property is a robust stability property in the sense that it is valid for an entire class of nonlinear functions - defined by (12) - and not for a specific one: in fact the stability conditions contain only information about the linear part and the sector where the nonlinear functions are confined - see (11) also (12).

B. Along its almost 70 years of history (the first reference is considered to be from 1942 - see the reference list of [17] two basic methods asserted themselves:

a) the direct method of Liapunov via a Liapunov function of the form "quadratic form plus integral of the nonlinear function"

$$
V(x)=x^{*} H x+\theta \int_{0}^{c^{*} x} \phi(\vartheta) \mathrm{d} \vartheta
$$

b) the method of the Popov like frequency domain inequality:

$$
\Re e Z(\imath \omega)\left(\frac{1}{\bar{\varphi}}+\left(1+\frac{\underline{\varphi}}{\bar{\varphi}}\right) \tilde{\kappa}(\imath \omega)+\bar{\varphi}|\tilde{\kappa}(\imath \omega)|^{2}\right) \geq 0,
$$

where $\tilde{\kappa}(\sigma)=c^{*}(\sigma I-A)^{-1} b$ is the transfer function of the linear part of (10) and the stability multiplier $Z(\sigma)$ is defined in the case of the standard Popov criterion by

$$
Z(\sigma)=\tau+\theta \sigma
$$

(The $\mathrm{P}$ (roportional) $\mathrm{D}$ (erivative) multiplier, see [15]).

It is now a well established fact that the two approaches are equivalent in the sense that finding of $H$ (Hermitian matrix) and $\theta$ (a real parameter) in (13) ensuring the required properties of $V$ requires solution of certain Linear Matrix 


\section{VLADIMIR RĂSVAN}

Inequalities. At their turn these Linear Matrix Inequalities can be solved if and only if (14) holds with $Z(\sigma)$ defined by (15).

The previous considerations indicate also the ways of extending these results to other classes of systems. The extensions may be performed in two ways. The first one concerns the extension of the class of linear blocks; the first step here is to consider time delay blocks of the form

$$
\dot{x}(t)=A_{0} x(t)+A_{1} x(t-\tau)+b \mu(t), \quad \nu=c^{*} x
$$

or even more general (several delays, distributed delays, delayed input, delayed output etc.). Since such a linear system can be given an integral form using the Cauchy formula of the variations of parameters, an even more general system than (16) would be an integral one, having one of the forms below

$$
\nu(t)=\rho(t)+\int_{0}^{t} \kappa(t-\tau) \mu(\tau) \mathrm{d} \tau ; \quad \nu(t)=\int_{-\infty}^{t} \kappa(t-\tau) \mu(\tau) \mathrm{d} \tau
$$

or even the "anticipative" (non-causal) system

$$
\nu(t)=\int_{-\infty}^{\infty} \kappa(t-\tau) \mu(\tau) \mathrm{d} \tau
$$

All these linear systems have in common one feature - the "continuous time", i.e., the independent variable $t \in \mathbb{R}$. But various applications such as computer control, digital signal modulation a.o. provide also discrete time systems such as

$$
x_{k+1}=A x_{k}+b \mu_{k}, \quad \nu=c^{*} x
$$

and its "integral" counterparts

$$
\nu_{k}=\rho_{k}+\sum_{0}^{k-1} \kappa_{k-1-i} \mu_{i}, \quad \nu_{k}=\sum_{-\infty}^{k-1} \kappa_{k-1-i} \mu_{i}, \quad \nu_{k}=\sum_{-\infty}^{\infty} \kappa_{k-1-i} \mu_{i} .
$$

It is exactly the discrete time case that offers a first motivation for restricting the class of nonlinear functions. Namely the inequality of (14) type can be obtained only for $Z(\sigma) \equiv$ const (the so called Tsypkin like criterion [14]); if the discrete analogue of (15) is required (the so called Jury-Lee criterion), then it is necessary to have $\phi(\nu)$ non decreasing [14].

Another motivation for considering additional restrictions for the nonlinear functions in the sector (12) has been suggested long ago in a paper of R. E. K a l m a n published in 1957 (see [14] for the exact reference): by making the class of sector restricted functions more narrow, it is possible to make the stability sector larger.

This idea deserves additional explanation. Among the functions confined in the sector we count the linear ones also. Were only these functions the ones 


\section{SLOPE RESTRICTED NONLINEARITIES}

for which absolute stability is required, the absolute stability would become a standard linear stability problem with a maximal stability sector $(\underline{\varphi}, \bar{\varphi})$. Consideration of nonlinear functions for the (global) stability requirement enlarges the class of sector restricted functions; while this would imply a more narrow stability sector, it has been conjectured by M. A. A i z e r m a n in 1948 (see, e.g., [8] ) that the absolute stability sector would coincide with the linear ("Hurwitz") one. This conjecture which holds for the first order systems, is disproved even for the second order ones in some special cases and there exists a celebrated counter-example of V. A. P lis s from 1958 which is of the third order. In the above mentioned paper Kalman conjectured that the linear and absolute stability sectors possibly coincide for sector and slope restricted nonlinearities (since the linear functions are both sector and slope restricted). The Kalman conjecture was also disproved but it turned to be true for all the third order systems while for larger order counter examples may be constructed in an algorithmic way [3].

As an outcome of the above issues, there were considered monotone, oddmonotone, globally Lipschitz and slope restricted nonlinear functions which were also sector confined.

C. Like in the discrete time case, the restriction of the admissible class of nonlinear functions allowed an extension of the class of multipliers $Z(\sigma)$. We give below some examples of such multipliers:

- the Brockett-Willems multiplier [9]

$$
Z_{B W}(\sigma)=\left(a_{0} \sigma+\sum_{1}^{m} a_{i} \frac{\sigma+z_{i}}{c_{i} \sigma+z_{i}}\right)^{ \pm 1}, \quad a_{i}>0, \quad z_{i}>0, \quad 0<c_{i} \leq 1,
$$

- the Yakubovich multiplier [23]

$$
Z_{Y}(\sigma)=1+\theta \sigma-\beta \sigma^{2},
$$

- the $\mathrm{P}$ (roportional) I(ntegral) multiplier [22]

$$
Z_{P I}(\sigma)=1+\theta(\sigma)^{-1}
$$

- the Baker-Desoer multiplier [2]

$$
Z_{B D}(\sigma)=1+\theta \sigma+\tilde{\varsigma}(\sigma),
$$

where $\tilde{\varsigma}(\sigma)$ is the Laplace transform of some non-positive function $\varsigma \in \mathcal{L}^{1}(0, \infty)$ and such that $1+\tilde{\varsigma}(0)>0$.

- the Zames-Falb multiplier [24]

$$
Z_{Z F}(\sigma)=\sum_{1}^{\infty} \zeta_{k} \mathrm{e}^{-\tau_{k} \sigma}+\int_{-\infty}^{\infty} \zeta(t) \mathrm{e}^{-\sigma t} \mathrm{~d} t
$$




\section{VLADIMIR RĂSVAN}

Remark that the last two multipliers may not be rational functions.

The stability inequalities for each type of multiplier are obtained under specific assumptions and with a specific design task: this means that a definite type of multiplier may be helpful in some cases and harmful in other ones. We do not insist on this matter but consider a definite case.

\section{The Yakubovich multiplier and its versions}

Our starting point will be system (10) with $\phi(\nu)$ subject to the sector inequalities (12). As we already mentioned in the previous section, obtaining a frequency domain stability inequality with some kind of multiplier requires some additional restriction on the class of nonlinear functions (the only exception seems to be the Popov multiplier; however the sector restriction (12) may be satisfied by some time varying nonlinear function but in this case there is no multiplier; therefore time invariance is a restriction for the Popov multiplier).

Therefore we shall consider the slope restriction

$$
\underline{\nu} \leq \phi^{\prime}(\nu) \leq \bar{\nu}
$$

and assume as in [23] that $\underline{\varphi}=0$. The frequency domain stability inequality is

$$
\begin{aligned}
\tau_{1}\left(\frac{1}{\bar{\varphi}}+\Re e \tilde{\kappa}(\imath \omega)\right) & +\tau_{2} \Re e \imath \omega \tilde{\kappa}(\imath \omega) \\
& +\tau_{3} \omega^{2} \Re e(1+\underline{\nu} \tilde{\kappa}(-\imath \omega))(1+\bar{\nu} \tilde{\kappa}(\imath \omega)) \geq 0
\end{aligned}
$$

for some real numbers $\tau_{i}$, where $\tau_{1} \geq 0, \tau_{3} \geq 0$. If this condition holds for $\tau_{1}=0$, we may multiply by $\omega^{-2}$ and with the choice $\tau_{3}=\bar{\nu}^{-1}$ the inequality (27) becomes

$$
\frac{1}{\bar{\nu}}+\Re e\left(1+\frac{\nu}{\bar{\nu}}-\frac{\tau_{2}}{\imath \omega}\right) \tilde{\kappa}(\imath \omega)+\left(\frac{\nu}{\bar{\nu}}\right)|\tilde{\kappa}(\imath \omega)|^{2} \geq 0
$$

Obviously, this frequency domain inequality incorporates only slope information about the nonlinear function. If additionally $\underline{\nu}=0$ in (26), then (28) may be written as

$$
\Re e\left(1-\frac{\tau_{2}}{\imath \omega}\right)\left(\frac{1}{\bar{\nu}}+\tilde{\kappa}(\imath \omega)\right) \geq 0
$$

thus obtaining the PI multiplier. Under this form the frequency domain inequality appears in [22], 12]. The same condition has been also reported in [5], i.e., earlier than [22]. We mention also that unlike [22] the paper [12] deals with several nonlinear elements; with respect to this it was preceded by [13], whose aim was to give more convincing proofs than in 22 but within the framework of several nonlinear elements and beyond the standard case with $A$, a Hurwitz matrix. 


\section{SLOPE RESTRICTED NONLINEARITIES}

If we leave aside these various frameworks (different sectors, single/several nonlinear function(s), non-Hurwitz matrix $A$ ) and focus on the standard framework (single nonlinear function, Hurwitz matrix $A, \underline{\varphi}=\underline{\nu}=0$ ), then the same frequency domain inequality (29) may be obtained, but under different additional (technical) assumptions. This might be considered just a matter of researcher's technique but it is in fact connected with a basic choice - the way of associating an augmented system. Without discussing the general analysis of [4], we shall describe briefly each case above.

In [23] the augmented system was defined by the state variables

$$
z=x, \quad \zeta=-\phi\left(c^{*} x\right)
$$

what sends to the $n+1$ dimensional system

$$
\begin{aligned}
& \dot{z}=A z+b \zeta, \\
& \dot{\zeta}=-\phi^{\prime}\left(c^{*} z\right) c^{*}(A z+b \zeta) .
\end{aligned}
$$

Obviously this system has the prime integral

$$
\zeta(t)+\phi\left(c^{*} z(t)\right) \equiv \text { const },
$$

hence its dimension may be reduced by one; moreover if the solutions of (131) are viewed on the invariant set $\zeta+\phi\left(c^{*} z\right) \equiv 0$ - suggested by $(30)$ - then $z(t) \equiv x(t)$ provided $z(0)=x(0)$. This extended system was considered in [7] for the case of several nonlinear functions.

Consider now the approach of [5]; here the new state variables are

$$
z=A x-b \phi\left(c^{*} x\right), \quad \zeta=-\phi\left(c^{*} x\right)
$$

and unlike (30) here $z=\dot{x}$. From here the following is obtained

$$
\begin{aligned}
& \dot{z}=A z+b \mu(t), \\
& \dot{\zeta}=\mu(t), \quad \mu(t)=-\phi^{\prime}\left(c^{*} x(t)\right) c^{*} z .
\end{aligned}
$$

If $\operatorname{det} A \neq 0$, then we may compute $c^{*} x=c^{*} A^{-1}(z-b \zeta)$ to obtain the $(n+1)-$ -dimensional system

$$
\begin{aligned}
& \dot{z}=A z+b \zeta, \\
& \dot{\zeta}=-\phi^{\prime}\left(c^{*} A^{-1}(z-b \zeta)\right) c^{*} z
\end{aligned}
$$

with the prime integral

$$
\zeta(t)+\phi\left(c^{*} A^{-1}(z(t)-b \zeta(t))\right) \equiv \text { const. }
$$

The third approach of [22, [12] is based on "differentiating the initial system"; this means,

hence

$$
z=A x-b \phi\left(c^{*} x\right), \quad \zeta=c^{*} x,
$$




$$
\begin{aligned}
& \text { VLADIMIR RĂSVAN } \\
& \dot{z}=A z-b \phi^{\prime}(\zeta) c^{*} z, \\
& \dot{\zeta}=c^{*} z
\end{aligned}
$$

with the prime integral

$$
\zeta(t)-c^{*} A^{-1} z(t)-c^{*} A^{-1} b \phi(\zeta(t)) \equiv \text { const. }
$$

Not only that (30) are the simplest in defining the new state variables but also the "return" to the basic system (10) via the associated prime integral generating a family of invariant sets is much simpler. This suggests, especially when thinking to the assumption $\operatorname{det} A \neq 0$ that slope restrictions are taken into account in a more natural way if considered together with the sector restrictions.

\section{Two frequency domain criteria for absolute stability: a comparison}

We shall state first the Yakubovich type criterion for the single nonlinearity case, in a very general framework for the sectors, slope restrictions and non-Hurwitz matrix $A$.

TheOREM 1. Consider the system (10) under the following assumptions: $i)$ it is non-degenerate, i.e., $(A, b)$ is a controllable pair and $\left(c^{*}, A\right)$ is an observable pair; ii) the $\mathcal{C}^{1}$-function $\phi: \mathbb{R} \mapsto \mathbb{R}$ is subject to the sector conditions (12) and slope restrictions (26); iii) the system is minimally stable, i.e., there exists a linear function $\phi(\nu)=\tilde{\varphi} \nu$ with $\tilde{\varphi} \in[\underline{\varphi}, \bar{\varphi}] \subseteq[\underline{\nu}, \bar{\nu}]$ such that $A-b \tilde{\varphi} c^{*}$ is a Hurwitz matrix.

Suppose there exist the real nonnegative numbers $\tau \geq 0, \beta \geq 0$ and the real number $\theta$ such that the following frequency domain stability inequality holds for all $\omega \geq 0$

$$
\begin{aligned}
\frac{\tau}{\bar{\varphi}}+\frac{\beta}{\bar{\nu}} \omega^{2} & +\Re e\left(\tau\left(1+\frac{\varphi}{\bar{\varphi}}\right)+\imath \omega \theta+\beta\left(1+\frac{\underline{\nu}}{\bar{\nu}}\right) \omega^{2}\right) \tilde{\kappa}(\imath \omega) \\
& +\left(\tau \underline{\varphi}+\beta \underline{\nu} \omega^{2}\right)|\tilde{\kappa}(\imath \omega)|^{2} \geq 0 .
\end{aligned}
$$

Moreover, the following alternative is valid. If $A$ is hyperbolic, i.e., it has no eigenvalues on the imaginary axis $\imath \mathbb{R}$, then (40) is strict including for $\omega \rightarrow \infty$ while the sector conditions (12) and the slope restrictions (26) are non strict; if $A$ has some eigenvalues on $\imath \mathbb{R}$, i.e., system (10) is in a critical case, then (40) is non strict while (12) and (26) are strict; also in this last case, if $\tau=0$, then $(1+\underline{\varphi} \tilde{\kappa}(0))(1+\bar{\varphi} \tilde{\kappa}(0))>0$. 


\section{SLOPE RESTRICTED NONLINEARITIES}

Assume also that the following condition is fulfilled

$$
\liminf _{\lambda \rightarrow \infty} \frac{\theta}{\lambda^{2}}\left(\int_{0}^{\lambda} \phi(\vartheta) \mathrm{d} \vartheta-\frac{1}{2} \lambda \phi(\lambda)\right) \geq 0 .
$$

Then system (10) is absolutely stable, i.e., its equilibrium at 0 is globally asymptotically stable for all functions in the corresponding admissible class, i.e., subject to (12), (26) and (41).

As we already mentioned, in the case of Theorem 1 the slope restrictions are taken into account in a more natural way, together with the sector restrictions. The proof of Theorem 1 follows from the main result of [20], where the case of several nonlinearities is considered, and will not be reproduced here. We shall point out however that this proof makes use of both sector and slope restrictions. Consequently, even if the sector information may be eliminated from the frequency domain inequality - see (28) - they remain present in defining the class of nonlinear functions for which absolute stability is valid.

We recall now the short note [22] where it had been stated that the proposed criterion (with PI multiplier) incorporated slope information only; this meant that this information only was present both in the frequency domain inequality and in defining the class of nonlinear functions for which absolute stability was valid (unlike the criterion of Theorem 1).

Since the proofs of [22] were far from convincing, an attempt was made in [13] to "fill the gap"; at the same time some generalizations were considered there: several nonlinear elements, unitary treatment of stable, critical and unstable cases, the set of prime integrals of the type (39). The stability criterion thus obtained displays however another drawback: the class of nonlinear functions depends on the numbers $\tilde{\varphi}_{i}-\tilde{\varphi}$ in the single nonlinearity case - ensuring minimal stability.

For this reason we shall reconsider here the main result of [13], more precisely the single nonlinearity case of [16] and eliminate the dependence on $\tilde{\varphi}$. The result reads as follows.

ThEOREM 2. Consider the system (10) under the following assumptions: $i)$ $\operatorname{det} A \neq 0,\left(c^{*}, A\right)$ is an observable pair and $c^{*} A^{-1} b \neq 0$; ii) the $\mathcal{C}^{1}$-function $\phi: \mathbb{R} \mapsto \mathbb{R}$ is subject to the slope restrictions (26); iii) the system is minimally stable, i.e., there exists a linear function $\phi(\nu)=\tilde{\varphi} \nu$ with $\tilde{\varphi} \in[\underline{\nu}, \bar{\nu}]$ such that $A-b \tilde{\varphi} c^{*}$ is a Hurwitz matrix.

Suppose there exist $\tau \geq 0$ and some real number $\theta$ such that the following frequency domain stability inequality holds for all $\omega \geq 0$

$$
\tau+\Re e\left(\tau(\underline{\nu}+\bar{\nu})-\frac{\theta}{\imath \omega}\right) \tilde{\kappa}(\imath \omega)+\tau \underline{\nu} \bar{\nu}|\tilde{\kappa}(\imath \omega)|^{2} \geq 0 .
$$




\section{VLADIMIR RĂSVAN}

Also the following alternative is valid. If $A$ is hyperbolic, i.e., it has no eigenvalues on the imaginary axis $\imath \mathbb{R}$, then (42) is strict including for $\omega \rightarrow \infty$ while the slope restrictions (26) are non strict; if $A$ has some eigenvalues on $\imath \mathbb{R}$, i.e., system (10) is in a critical case, then (42) is non strict while (26) are strict; also, if in both cases the Left Hand Side of (42) is identical to a constant or in the last case, $\tau=0$, then $(1+\underline{\nu} \tilde{\kappa}(0))(1+\bar{\nu} \tilde{\kappa}(0))>0$. Assume also that (41) is fulfilled. Then system (10) is absolutely stable, i.e., its equilibrium at 0 is globally asymptotically stable for all functions in the corresponding admissible class, i.e., subject to (26) and (41).

The two theorems have a lot in common but it is worth mentioning some differences. The first assumption is rather technical: if we want to eliminate the controllability assumption on $(A, b)$, then a certain necessary assumption on the uncontrollable spectrum in order to apply the Yakubovich-Kalman-Popov lemma will prevent $A$ to have no zero eigenvalue. Also the condition $c^{*} A^{-1} b=\tilde{\kappa}(0) \neq 0$ is technical, i.e., it is imposed by the proof technique.

Due to the novelty of this result with respect to [13, [16] - in fact it is a genuine improvement - a sufficiently detailed proof is given in the Appendix.

\section{Some examples}

A. We shall borrow an application from [7. Consider the third order differential equation

$$
y^{\prime \prime \prime}+\phi\left(y^{\prime \prime}\right)+\alpha_{1} y^{\prime}+\alpha_{0} y=0 ; \quad \alpha_{0}>0, \quad \alpha_{1}>0
$$

where $\phi: \mathbb{R} \mapsto \mathbb{R}$ is a $\mathcal{C}^{1}$ function. We want to obtain the maximal sector and/or the maximal slope restriction domain, i.e., to solve the so called Kalman problem.

If $\phi(\nu)=\alpha_{2} \nu$ then the Hurwitz condition reads $\alpha_{2}>\alpha_{0} / \alpha_{1}$. Let $\phi(\nu)=$ $\left(\alpha_{0} / \alpha_{1}\right) \nu+\psi(\nu)$; system (43) becomes

$$
y^{\prime \prime \prime}+\left(\alpha_{0} / \alpha_{1}\right) y^{\prime \prime}+\psi\left(y^{\prime \prime}\right)+\alpha_{1} y^{\prime}+\alpha_{0} y=0
$$

which may be written as (10) with

$$
A=\left(\begin{array}{ccc}
0 & 1 & 0 \\
0 & 0 & 1 \\
-\alpha_{0} & -\alpha_{1} & -\alpha_{0} / \alpha_{1}
\end{array}\right), \quad b=\left(\begin{array}{l}
0 \\
0 \\
1
\end{array}\right), \quad c^{*}=\left(\begin{array}{lll}
0 & 0 & 1
\end{array}\right) .
$$

The transfer function of the linear part is

$$
\tilde{\kappa}(\sigma)=\frac{\sigma^{2}}{\sigma^{3}+\left(\alpha_{0} / \alpha_{1}\right) \sigma^{2}+\alpha_{1} \sigma+\alpha_{0}}=\frac{\sigma^{2}}{\left(\sigma+\alpha_{0} / \alpha_{1}\right)\left(\sigma^{2}+\alpha_{1}\right)} .
$$




\section{SLOPE RESTRICTED NONLINEARITIES}

Since $\tilde{\kappa}(0)=0$ only Theorem 1 can be applied. Due to the condition $\alpha_{2}>\alpha_{0} / \alpha_{1}$ the Hurwitz sector for (44) is $(0, \infty)$. The frequency domain inequality is deduced from (40) as below

$$
\frac{\tau}{\bar{\varphi}}+\frac{\beta}{\bar{\nu}} \omega^{2}-\Re e\left(\tau+\beta \omega^{2}+\imath \omega \theta\right) \frac{\omega^{2}}{\left(\alpha_{1}-\omega^{2}\right)\left(\left(\alpha_{0} / \alpha_{1}\right)+\imath \omega\right)} \geq 0 .
$$

Without any loss of generality we may choose $\tau=1$. The choice $\theta=-(1+$ $\left.\alpha_{1} \beta\right)\left(\alpha_{0} / \alpha_{1}^{2}\right)$ is compulsory to simplify $\alpha_{1}-\omega^{2}$ which might otherwise induce a change of sign at $\omega=\sqrt{\alpha_{1}}$. It follows that

$$
\frac{1}{\bar{\varphi}}+\frac{\beta}{\bar{\nu}}-\frac{\alpha_{0}}{\alpha_{1}^{2}} \frac{\omega^{2}}{\left(\alpha_{0} / \alpha_{1}\right)^{2}+\omega^{2}} \geq 0
$$

which gives

$$
\frac{\beta}{\bar{\nu}} \lambda^{2}+\left(\frac{1}{\bar{\varphi}}+\frac{\beta}{\bar{\nu}}\left(\frac{\alpha_{0}}{\alpha_{1}}\right)^{2}-\frac{\alpha_{0}}{\alpha_{1}^{2}}\right) \lambda+\frac{1}{\bar{\varphi}}\left(\frac{\alpha_{0}}{\alpha_{1}}\right)^{2} \geq 0 .
$$

Obviously the final choice $\beta / \bar{\nu}>1 / \alpha_{0}$ ensures fulfilment of the above inequality for $\bar{\varphi}>0$ and $\bar{\nu}>0$ arbitrarily large (but nevertheless finite).

B. Consider now the third order differential equation [6], 18]

$$
y^{\prime \prime \prime}+\alpha_{2} y^{\prime \prime}+\alpha_{1} y^{\prime}+\alpha_{0} y+f(y)=0
$$

where $f: \mathbb{R} \mapsto \mathbb{R}$ is again a $\mathcal{C}^{1}$-function, $\alpha_{2}>0, \alpha_{0}>0, \alpha_{1} \alpha_{2}>\alpha_{0}$. Re-writing the system by introducing $-\phi(\nu)=\left(\alpha_{0}-\alpha_{1} \alpha_{2}\right) \nu+f(\nu)$ we obtain

$$
y^{\prime \prime \prime}+\alpha_{2} y^{\prime \prime}+\alpha_{1} y^{\prime}+\alpha_{1} \alpha_{2} y-\phi(y)=0
$$

which may be written as (10) with

$$
A=\left(\begin{array}{ccc}
0 & 1 & 0 \\
0 & 0 & 1 \\
-\alpha_{1} \alpha_{2} & -\alpha_{1} & -\alpha_{2}
\end{array}\right), \quad b=\left(\begin{array}{c}
0 \\
0 \\
-1
\end{array}\right), \quad c^{*}=\left(\begin{array}{lll}
1 & 0 & 0
\end{array}\right) .
$$

The transfer function of the linear part is

$$
\tilde{\kappa}(\sigma)=\frac{-1}{\left(\sigma^{2}+\alpha_{1}\right)\left(\sigma+\alpha_{2}\right)}, \quad \tilde{\kappa}(0)=\frac{-1}{\alpha_{1} \alpha_{2}}<0
$$

and we may apply Theorem 2 in the critical case with $\underline{\nu}=0, \tau=1 / \bar{\nu}$ :

$$
\frac{1}{\bar{\nu}}+\Re e\left(1-\frac{\theta}{\imath \omega}\right) \frac{\alpha_{2}-\imath \omega}{\left(\alpha_{1}-\omega^{2}\right)\left(\alpha_{2}^{2}+\omega^{2}\right)}=\frac{1}{\bar{\nu}}+\frac{\alpha_{2}+\theta}{\left(\alpha_{1}-\omega^{2}\right)\left(\alpha_{2}^{2}+\omega^{2}\right)} \geq 0
$$

and the only choice to simplify $\alpha_{1}-\omega^{2}$ is $\theta=-\alpha_{2}<0$; in this way the LHS of (49) reduces to a strictly positive constant. The additional condition of Theorem 2 in this case will give $\bar{\nu}<\alpha_{1} \alpha_{2}$. Stability is thus ensured for any $\phi(y)$ satisfying $0<\phi^{\prime}(y)<\alpha_{1} \alpha_{2}$ hence for any $f(y)$ subject to $-\alpha_{0}<f^{\prime}(y)<\alpha_{1} \alpha_{2}-\alpha_{0}$ and 


\section{VLADIMIR RĂSVAN}

to (41) in the case $\theta<0$. In this way a result of [21, Satz 4.15, p. 320] even with a slight generalization since now we have $\alpha_{0} \neq 0$ unlike in [21].

C. Consider now the fourth order differential equation

$$
y^{\prime \prime \prime \prime}+\alpha y^{\prime \prime \prime}+\beta y^{\prime \prime}+\gamma y^{\prime}+f(y)=0, \quad \alpha>0, \quad \alpha \beta-\gamma>0 .
$$

The Hurwitz conditions for the fourth degree polynomial

are

$$
p(\lambda) \equiv \lambda^{4}+\alpha \lambda^{3}+\beta \lambda^{2}+\gamma \lambda+\delta
$$

hence $\beta>0, \gamma>0$. Introducing

we obtain

$$
\phi(\nu)=\left(\beta \gamma / \alpha-(\gamma / \alpha)^{2}\right) \nu-f(\nu)
$$

$$
y^{\prime \prime \prime \prime}+\alpha y^{\prime \prime \prime}+\beta y^{\prime \prime}+\gamma y^{\prime}+\left(\beta \gamma / \alpha-(\gamma / \alpha)^{2}\right) y-\phi(y)=0 .
$$

This equation may be given the form (10) with

$$
A=\left(\begin{array}{cccc}
0 & 1 & 0 & 0 \\
0 & 0 & 1 & 0 \\
0 & 0 & 0 & 1 \\
-\left(\beta \gamma / \alpha-(\gamma / \alpha)^{2}\right) & -\gamma & -\beta & -\alpha
\end{array}\right), \quad b=\left(\begin{array}{c}
0 \\
0 \\
0 \\
-1
\end{array}\right), \quad c^{*}=\left(\begin{array}{llll}
1 & 0 & 0 & 0
\end{array}\right)
$$

and the transfer function

$$
\tilde{\kappa}(\sigma)=\frac{-\alpha^{2}}{\left(\alpha \sigma^{2}+\gamma\right)\left(\alpha \sigma^{2}+\alpha^{2} \sigma+\alpha \beta-\gamma\right)}
$$

having two poles on $\imath \mathbb{R}$ and two other in $\mathbb{C}^{-}$. We may apply again Theorem 2 in the critical case with $\underline{\nu}=0, \tau=1 / \bar{\nu}$. After some tedious manipulation that we do not reproduce here we find the unique choice $\theta=(2 \gamma-\alpha \beta) \alpha^{-2}$ to simplify the factor $\left.\gamma-\alpha \omega^{2}\right)$ that might produce a change of sign and to obtain finally

$$
\alpha^{2} \bar{\nu} \leq \min _{\lambda \geq 0}\left[(\alpha \beta-\gamma-\alpha \lambda)^{2}+\alpha \lambda\right]
$$

We do not reproduce the final form of the maximally large slope restrictions since this is just a matter of elementary manipulation.

\section{Some conclusions and future challenge}

Throughout the paper a critical analysis has been performed concerning the role of certain stability multipliers that are introduced when slope restrictions for the nonlinear functions are taken into account. Only the single nonlinearity case was considered. The main result of the paper appears to be Theorem 2 where some non-negligible errors of the previous papers [13, [16, [18, have been corrected. Consequently, the results concerning some applications of [18] were 


\section{SLOPE RESTRICTED NONLINEARITIES}

sensibly improved. The future research on this type of multiplier arising from the idea of considering the slope information only aims to the case of several nonlinear elements and to the extension to the case of the integral equations. The improvement of this stability criterion for the case of several nonlinear elements should provide an improvement on the conditions of a "good" qualitative behavior for such important applications as neural networks (see [19]).

\section{REFERENCES}

[1] ANONIMOUS: Flight Control Design—Best Practices. NATO-RTO Technical Report 29, December 2000.

[2] BAKER, R. A.-DESOER, C. A.: Asymptotic stability in the large of a class of single-loop feedback systems, SIAM J. Control 6 (1968), 1-8.

[3] BARABANOV, N. E.: About the problem of Kalman, Sibirsk. Math. Zh. XXIX (1988), 3-11. (In Russian)

[4] BARABANOV, N. E.: The state space extension method in the theory of absolute stability, IEEE Trans. Automat. Control 45 (2000), 2335-2339.

[5] BARABANOV, N. E.-YAKUBOVICH, V. A.: Absolute stability of control systems having one hysteresis-like nonlinearity, Avtomat. i Telemekh. 43 (1979), 5-12. (In Russian)

[6] BARBĂLAT, I.-HALANAY, A.: Nouvelles applications de la méthode fréquentielle dans la théorie des oscillations, Rev. Roum. Sci. Tech. Sér. Electrotechn. et Energ. 16 (1971), 689-702.

[7] BARBĂLAT, I.-HALANAY, A.: Conditions de comportement "presque linéaire" dans la théorie des oscillations, Rev. Roum. Sci. Tech. Sér. Electrotechn. et Energ. 19 (1974), 321-341.

[8] BARBAŠIN, E. A.: Introduction to Stability Theory. "Nauka" Publ. House, Moscow, 1967. (In Russian)

[9] BROCKETT, R. W.-WILLEMS, J. L.: Frequency domain stability criteria I, II, IEEE Trans. Automat. Control AC-10 (1965), 255-261, 407-413.

[10] GELIG, A. KH.-LEONOV, G. A.-YAKUBOVICH, V. A.: Stability of Nonlinear Systems with Nonunique Equilibrium State. "Nauka" Publ. House, Moscow, 1978. (In Russian)

[11] HADDAD, W. M.: Correction to "Absolute stability criteria for multiple slope-restricted monotonic nonlinearities", IEEE Trans. Automat. Control 42 (1997), 591.

[12] HADDAD, W. M.-KAPILA, V.: Absolute stability criteria for multiple slope-restricted monotonic nonlinearities, IEEE Trans. Automat. Control 40 (1995), 361-365.

[13] HALANAY, A.-RĂSVAN, VL.: Absolute stability of feedback systems with several differentiable non-linearities, Int. J. Systems Sci. 22 (1991), 1911-1927.

[14] HALANAY, A.-RĂSVAN, VL.: Stability and Stable Oscillations in Discrete Time Systems. Gordon and Breach Science Publ., Amsterdam, 2000.

[15] POPOV, V. M.: Hyperstability of Control Systems. Editura Academiei, Bucharest, 1966 (In Romanian); Russian improved version by Nauka Publ. House, Moscow, 1970; French improved version by Dunod, Paris, 1973; English improved version by Springer-Verlag, Berlin, 1973.

[16] RĂSVAN, VL.: New results and applications of the frequency domain criteria to absolute stability of nonlinear systems, in: Proc. Colloq. Qual. Theory Differ. Equ., Vol. 53, Szeged, Hungary, 1988, pp. 577-594. 


\section{VLADIMIR RĂSVAN}

[17] RĂSVAN, VL.: Delay-independent and delay-dependent Aizerman problem, in: Unsolved Problems in Mathematical Systems and Control Theory (V. D. Blondel and A. Megretski, eds.), Princeton University Press, Princeton and Oxford, 2002, pp. 212-220.

[18] RĂSVAN, VL.: A new dissipativity criterion-Towards Yakubovich oscillations, Internat. J. Robust Nonlinear Control 17 (2007), 483-495.

[19] RĂSVAN, VL.-DANCIU, D.: On Popov-type stability criteria for neural networks, in: Proc. of the 6'th Coll. Qualitative Theory of Diff. Equ., Electron. J. Qual. Theory Differ. Equ. Vol. 23, 2000, pp. 1-10, http://www.math.u-szeged.hu/ejqtde/6/623.html .

[20] RĂSVAN, VL.-DANCIU, D.-POPESCU, D.: On absolute (robust) stability: slope restrictions and stability multipliers, Int. J. Robust Nonlin. Contr. (submitted).

[21] REISSIG, R.-SANSONE, G.-CONTI, R.: Nichtlineare Differentialgleichungen höhere Ordnung. Edizioni Cremonese, Rome, 1969.

[22] SINGH, V.: A stability inequality for nonlinear feedback systems with slope-restricted nonlinearity, IEEE Trans. Automat. Control 29 (1984), 743-744.

[23] YAKUBOVICH, V. A.: The method of the matrix inequalities in the the theory of stability for nonlinear controlled systems II. Absolute stability in the class of slope restricted nonlinearities, Avtomat. i Telemekh. XXIX (1965), 577-590. (In Russian)

[24] ZAMES, G.-FALB, P. L.: Stability conditions for systems with monotone and slope-restricted nonlinearities, SIAM J. Control 6 (1968), 89-108.

\section{Appendix}

\section{Proof of Theorem 2}

The proof will follow the line of [7], 20], i.e., combining the methods of positiveness theory [15] and of the Liapunov second method for robustness proofs (i.e., ensuring stability for an entire class of systems containing some uncertainty).

We start from system (10) and assume $\phi: \mathbb{R} \mapsto \mathbb{R}$ being $\mathcal{C}^{1}$ and satisfying (26). Introducing new state variables

$$
z=A x-b \phi\left(c^{*} x\right), \quad \eta=c^{*} x,
$$

they satisfy the following $(n+1)$-dimensional system

$$
\dot{z}=A z-b \phi^{\prime}(\eta) c^{*} z, \quad \dot{\eta}=c^{*} z .
$$

This system has the prime integral

$$
\eta(t)-\left(c^{*} A^{-1} b\right) \phi(\eta(t))-c^{*} A^{-1} z(t) \equiv \mathrm{const}
$$

and, if $\eta(t)$ and $z(t)$ are constructed starting from a solution of (10), the constant in (55) is 0 .

A. We associate to (54) the so called P o pov system [15] composed of the control system

$$
\left(\begin{array}{c}
\dot{z} \\
\dot{\eta}
\end{array}\right)=\left(\begin{array}{cc}
A & 0 \\
c^{*} & 0
\end{array}\right)\left(\begin{array}{l}
z \\
\eta
\end{array}\right)+\left(\begin{array}{l}
b \\
0
\end{array}\right) \mu(t)
$$




\section{SLOPE RESTRICTED NONLINEARITIES}

and the integral index

$$
\chi^{o}(0, t)=\int_{0}^{t} \mathcal{F}^{o}(\mu(\vartheta), z(\vartheta), \eta(\vartheta)) \mathrm{d} \vartheta
$$

where the quadratic form $\mathcal{F}^{o}(\mu, z, \eta)$ is as follows

$$
\mathcal{F}^{o}(\mu, z, \eta)=\tau\left(\mu+\underline{\nu} c^{*} z\right)\left(\mu+\bar{\nu} c^{*} z\right)-\theta \mu \eta \text {. }
$$

Here $\tau \geq 0$ and $\theta \in \mathbb{R}$ are those of the frequency domain inequality (42) of Theorem 2, Following [15] we shall consider (56)-(58) as having complex coefficients $A, b, c$ and complex arguments $\mu, z, \eta$. Therefore $\mathcal{F}^{o}$ is extended to complex values of its arguments as follows

$$
\begin{aligned}
\mathcal{F}^{o}(\mu, z, \eta)= & \tau \bar{\mu} \mu+\frac{1}{2} \tau(\underline{\nu}+\bar{\nu})\left(\bar{\mu} c^{*} z+z^{*} c \mu\right) \\
& +\tau \underline{\nu} \bar{\nu} z^{*} c c^{*} z+\frac{1}{2} \theta(\bar{\mu} \eta+\bar{\eta} \mu)
\end{aligned}
$$

and the asterisk denotes transpose and complex conjugation or just transpose in the case of vectors and matrices with real entries.

Following [15] we associate to (56) and (59) the characteristic function of two complex variables

$$
\begin{aligned}
\tilde{\chi}^{o}(\lambda, \sigma)= & \tau+\frac{1}{2}(\tau(\underline{\nu}+\bar{\nu})+\theta / \sigma) \tilde{\kappa}(\sigma) \\
& +\frac{1}{2} \overline{\tilde{\kappa}}(\lambda)(\tau(\underline{\nu}+\bar{\nu})+\theta / \lambda)+\tau \underline{\nu} \bar{\nu} \overline{\tilde{\kappa}}(\lambda) \tilde{\kappa}(\sigma),
\end{aligned}
$$

where $\tilde{\kappa}(\sigma)=c^{*}(\sigma I-A)^{-1} b$ is the transfer function of the linear block in (11) and $\overline{\tilde{\kappa}}(\lambda)=\overline{\tilde{\kappa}(\bar{\lambda})}$.

It has also to be mentioned that if $\bar{\nu}=\infty$, then

$$
\mathcal{F}^{o}(\mu, z, \eta)=\tau\left(\mu+\underline{\nu} c^{*} z\right) c^{*} z-\theta \mu \eta
$$

in (58) and if $\underline{\nu}=-\infty$, then

$$
\mathcal{F}^{o}(\mu, z, \eta)=-\tau\left(\mu+\bar{\nu} c^{*} z\right) c^{*} z-\theta \mu \eta \text {. }
$$

Let (42) hold for some $\tau \geq 0, \theta \in \mathbb{R}$. It is easily seen that the LHS of (42) is exactly $\tilde{\chi}^{o}(-\imath \omega, \imath \omega)$, hence we have $\tilde{\chi}^{o}(-\imath \omega, \imath \omega) \geq 0$. We are thus in position to apply positiveness theorem [15, Chapter 3], for single input systems. According to this theorem, if $\tilde{\chi}^{o}(-\imath \omega, \imath \omega) \geq 0$, then there exist a scalar $\gamma^{o}$, a $n$-vector $w^{o}$ and a Hermitian $n \times n$ matrix $H^{o}$ such that along the solutions of (56), (57), 
(59) the following expression of $\chi^{o}(0, t)$ is valid

$$
\begin{aligned}
\chi^{o}(0, t)= & {\left[z^{*}(\vartheta) H^{o} z(\vartheta)-\frac{\theta}{c^{*} A^{-1} b}\left(z^{*}(\vartheta)\left(A^{*}\right)^{-1} c \eta(\vartheta)\right.\right.} \\
& \left.\left.+\bar{\eta}(\vartheta) c^{*} A^{-1} z(\vartheta)-2 \bar{\eta}(\vartheta) \eta(\vartheta)\right)\right]\left.\right|_{0} ^{t} \\
& +\int_{0}^{t}\left|\gamma^{o} \mu(\vartheta)+\left(w^{o}\right)^{*} z(\vartheta)\right|^{2} \mathrm{~d} \vartheta .
\end{aligned}
$$

(From the conditions of the problem it follows that since $\theta$ is real, $c^{*} A^{-1} b$ must be such).

If (42) is strict ( $>0$ ) including $\omega \rightarrow \infty$, then there will exist some $\varepsilon>0$ sufficiently small such that

$$
\tilde{\chi}^{\varepsilon}=\tilde{\chi}^{o}(-\imath \omega, \imath \omega)-\varepsilon b^{*}\left(-\imath \omega I-A^{*}\right)^{-1}(\imath \omega I-A)^{-1} b \geq 0 .
$$

Thus the positiveness theorem may be applied to a modified Popov system with

$$
\mathcal{F}^{\varepsilon}(\mu, z, \eta)=\mathcal{F}^{o}(\mu, z, \eta)-\varepsilon z^{*} z
$$

to find a scalar $\gamma^{\varepsilon}$, a $n$-vector $w^{\varepsilon}$ and a $n \times n$ hermitian matrix $H^{\varepsilon}$ such that

$$
\begin{aligned}
\chi^{\varepsilon}(0, t)= & {\left[z^{*}(\vartheta) H^{\varepsilon} z(\vartheta)-\frac{\theta}{c^{*} A^{-1} b}\left(z^{*}(\vartheta)\left(A^{*}\right)^{-1} c \eta(\vartheta)\right.\right.} \\
& \left.\left.+\bar{\eta}(\vartheta) c^{*} A^{-1} z(\vartheta)-2 \bar{\eta}(\vartheta) \eta(\vartheta)\right)\right]\left.\right|_{0} ^{t} \\
& +\int_{0}^{t}\left|\gamma^{\varepsilon} \mu(\vartheta)+\left(w^{\varepsilon}\right)^{*} z(\vartheta)\right|^{2} \mathrm{~d} \vartheta
\end{aligned}
$$

It is worth mentioning that if the coefficients $A, b, c$ are real, then $\gamma^{o}, w^{o}, H^{o}$ and $\gamma^{\varepsilon}, w^{\varepsilon}, H^{\varepsilon}$, respectively, may be chosen with real entries.

B. We shall write now the second form of $\chi^{\varepsilon}(0, t)$ and $\chi^{o}(0, t)$, respectively. Let $\hat{x}(t)$ be some solution of (10). Starting from this solution we define $\hat{z}(t)$ and $\hat{\eta}(t)$ in (53); these functions will verify (54) and also (55) with the constant equal to 0 , as already mentioned. Choosing $\mu(t)=-\phi^{\prime}(\hat{\eta}(t)) c^{*} \hat{z}(t)$ in (56), the solution $(z(t), \eta(t))$ of (56) will coincide with $\hat{z}(t), \hat{\eta}(t)$ and this is valid for any 


\section{SLOPE RESTRICTED NONLINEARITIES}

solution of (10). We may thus drop the "hat" and obtain

$$
\begin{aligned}
\chi^{\varepsilon}(0, t)= & \tau \int_{0}^{t}\left(\underline{\nu}-\phi^{\prime}(\eta(\vartheta))\right)\left(\bar{\nu}-\phi^{\prime}(\eta(\vartheta))\right)\left(c^{*} z(\vartheta)\right)^{2} \mathrm{~d} \vartheta \\
& -\theta \int_{\eta(0)}^{\eta(t)} \lambda \phi^{\prime}(\lambda) \mathrm{d} \lambda-\varepsilon \int_{0}^{t}|z(\vartheta)|^{2} \mathrm{~d} \vartheta
\end{aligned}
$$

The form of (65) shows that this part of the proof is done under the assumption that $A, b, c$ have real entries. The writing of $\chi^{o}(0, t)$ follows from (65) by letting $\varepsilon \rightarrow 0$.

Now we take the following procedure: take in (64) $\mu(\vartheta)=-\phi^{\prime}(\eta(\vartheta)) c^{*} z(\vartheta)$ and equate (64) and (65). Taking into account (55) - with zero constant - we find after some simple manipulation:

$$
\begin{gathered}
\left.z^{*}(\vartheta) H^{\varepsilon} z(\vartheta)\right|_{0} ^{t}+\int_{0}^{t}\left|-\gamma^{\varepsilon} \phi^{\prime}(\eta(\vartheta)) c^{*} z(\vartheta)+\left(w^{\varepsilon}\right)^{*} z(\vartheta)\right|^{2} \mathrm{~d} \vartheta \\
=\tau \int_{0}^{t}\left(\underline{\nu}-\phi^{\prime}(\eta(\vartheta))\right)\left(\bar{\nu}-\phi^{\prime}(\eta(\vartheta))\right)\left(c^{*} z(\vartheta)\right)^{2} \mathrm{~d} \vartheta \\
-\theta \int_{\eta(0)}^{\eta(t)} \phi(\lambda) \mathrm{d} \lambda-\varepsilon \int_{0}^{t}|z(\vartheta)|^{2} \mathrm{~d} \vartheta .
\end{gathered}
$$

This suggests the following state function

$$
\mathcal{V}^{\varepsilon}(z, \eta)=z^{*} H^{\varepsilon} z+\theta \int_{0}^{\eta} \phi(\lambda) \mathrm{d} \lambda .
$$

Along the solutions of (54) constructed starting from the solutions of (11) this function is subject to

$$
\begin{aligned}
\mathcal{V}^{\varepsilon}(z(t), \eta(t)) & \\
= & \mathcal{V}^{\varepsilon}(z(0), \eta(0))+\tau \int_{0}^{t}\left(\underline{\nu}-\phi^{\prime}(\eta(\vartheta))\right)\left(\bar{\nu}-\phi^{\prime}(\eta(\vartheta))\right)\left(c^{*} z(\vartheta)\right)^{2} \mathrm{~d} \vartheta \\
& -\int_{0}^{t}\left|-\gamma^{\varepsilon} \phi^{\prime}(\eta(\vartheta)) c^{*} z(\vartheta)+\left(w^{\varepsilon}\right)^{*} z(\vartheta)\right|^{2} \mathrm{~d} \vartheta-\varepsilon \int_{0}^{t}|z(\vartheta)|^{2} \mathrm{~d} \vartheta
\end{aligned}
$$


If the frequency domain inequality (42) is non-strict $(\geq 0)$, equality (68) takes the form arising from letting $\varepsilon \rightarrow 0$. Previous considerations suggest the state function

$$
\mathcal{V}^{o}(z, \eta)=z^{*} H^{o} z+\theta \int_{0}^{\eta} \phi(\lambda) \mathrm{d} \lambda
$$

subject to

$$
\begin{aligned}
\mathcal{V}^{o}(z(t), \eta(t)) & \\
= & \mathcal{V}^{o}(z(0), \eta(0))+\tau \int_{0}^{t}\left(\underline{\nu}-\phi^{\prime}(\eta(\vartheta))\right)\left(\bar{\nu}-\phi^{\prime}(\eta(\vartheta))\right)\left(c^{*} z(\vartheta)\right)^{2} \mathrm{~d} \vartheta \\
& -\int_{0}^{t}\left|-\gamma^{o} \phi^{\prime}(\eta(\vartheta)) c^{*} z(\vartheta)+\left(w^{o}\right)^{*} z(\vartheta)\right|^{2} \mathrm{~d} \vartheta-\varepsilon \int_{0}^{t}|z(\vartheta)|^{2} \mathrm{~d} \vartheta .
\end{aligned}
$$

The state functions $\mathcal{V}^{\varepsilon}$ and $\mathcal{V}^{o}$ are candidate Liapunov functions if considered along the solutions of (10), i.e., by making use of (53). We shall have first from (68)

$$
\frac{\mathrm{d}}{\mathrm{d} t} \mathcal{V}^{\varepsilon}\left(A x(t)-b \phi\left(c^{*} x(t)\right), c^{*} x(t)\right) \leq-\varepsilon\left|A x(t)-b \phi\left(c^{*} x(t)\right)\right|^{2}
$$

hence the derivative of $\mathcal{V}^{\varepsilon}$ along the solutions of (10) vanishes on the equilibria set only-here the singleton $\{0\}$.

In the second case the derivative of $\mathcal{V}^{o}$ along the solutions of (10) vanishes surely on the set where $c^{*} z=0$ hence for $\eta=$ const, $\dot{z}=A z$. Since $\left(c^{*}, A\right)$ is an observable pair, it follows that the derivative of $\mathcal{V}^{o}$ along the solutions of (10) also vanishes on the equilibria set of (10) - here the singleton $\{0\}$.

D. We search now a sign condition for the candidate Liapunov function itself. Let $\phi(\nu)=\tilde{\varphi} \nu$ where $\tilde{\varphi}$ is that of Theorem 2 hence $A-b \tilde{\varphi} c^{*}$ is a Hurwitz and, therefore, non-singular matrix. For this case the candidate Liapunov function becomes a quadratic form

$$
\mathcal{V}^{\varepsilon}\left(\left(A-b \tilde{\varphi} c^{*}\right) x, c^{*} x\right):=\mathcal{W}_{\tilde{\varphi}}^{\varepsilon}(x)=x^{*}\left[\left(A-b \tilde{\varphi} c^{*}\right)^{*} H^{\varepsilon}\left(A-b \tilde{\varphi} c^{*}\right)+\frac{1}{2} \theta \tilde{\varphi} c c^{*}\right] x
$$

and since $A_{\tilde{\varphi}}=A-b \tilde{\varphi} c^{*}$ is nonsingular, the derivative

$$
\frac{\mathrm{d}}{\mathrm{d} t} \mathcal{W}_{\tilde{\varphi}}^{\varepsilon}(x(t)) \leq-\varepsilon\left|A_{\tilde{\varphi}} x(t)\right|^{2}
$$

is negative definite. System (10) with $\phi(\nu)=\tilde{\varphi} \nu$ being exponentially stable, $\mathcal{W}_{\tilde{\varphi}}^{\varepsilon}(x)$ results positive definite hence

$$
\mathcal{W}_{\tilde{\varphi}}^{\varepsilon}(x) \geq \delta^{\varepsilon}(\tilde{\varphi})|x|^{2} .
$$




\section{SLOPE RESTRICTED NONLINEARITIES}

In the other case, of non-strict frequency domain inequality and strict slope restrictions we have $\underline{\nu}<\tilde{\varphi}<\bar{\nu}$ and

$$
\frac{\mathrm{d}}{\mathrm{d} t} \mathcal{W}_{\tilde{\varphi}}^{o}(x(t)) \leq \tau(\underline{\nu}-\tilde{\varphi})(\bar{\nu}-\tilde{\varphi})\left(c^{*} A_{\tilde{\varphi}} x(t)\right)^{2} .
$$

Since $\left(c^{*}, A\right)$ is an observable pair, $\left(c^{*}, A_{\tilde{\varphi}}\right)$ is such. Therefore we have in this case also

$$
\mathcal{W}_{\tilde{\varphi}}^{o}(x) \geq \delta^{o}(\tilde{\varphi})|x|^{2}
$$

because (10) is exponentially stable for $\phi(\nu)=\tilde{\varphi} \nu$.

The next step is to prove the same property for any $\phi(\nu)=\varphi \nu$, where $\varphi$ is arbitrary in the interval $[\underline{\nu}, \bar{\nu}]$ or $(\underline{\nu}, \bar{\nu})$. With respect to this we shall considered the "transformed" system

$$
\dot{x}=A_{\tilde{\varphi}} x-\left(\phi\left(c^{*} x\right)-\tilde{\varphi} c^{*} x\right),
$$

the associated system

$$
\dot{z}=A_{\tilde{\varphi}} z-b\left(\phi^{\prime}(\eta)-\tilde{\varphi}\right) c^{*} z, \quad \dot{\eta}=c^{*} z
$$

with its prime integral, the associated Popov controlled system

$$
\left(\begin{array}{c}
\dot{z} \\
\dot{\eta}
\end{array}\right)=\left(\begin{array}{cc}
A_{\tilde{\varphi}} & 0 \\
c^{*} & 0
\end{array}\right)\left(\begin{array}{l}
z \\
\eta
\end{array}\right)+\left(\begin{array}{l}
b \\
0
\end{array}\right) \mu(t)
$$

and the integral index

$$
\chi_{\tilde{\varphi}}^{o}(0, t)=\int_{0}^{t} \mathcal{F}_{\tilde{\varphi}}^{o}(\mu(\vartheta), z(\vartheta), \eta(\vartheta)) \mathrm{d} \vartheta
$$

with its quadratic form

$$
\mathcal{F}_{\tilde{\varphi}}^{o}(\mu, z, \eta)=\tau\left(\mu+(\underline{\nu}-\tilde{\varphi}) c^{*} z\right)\left(\mu+(\bar{\nu}-\tilde{\varphi}) c^{*} z\right)-\theta \mu \eta .
$$

The frequency domain characteristic will be

$$
\tilde{\chi}_{\tilde{\varphi}}^{o}(-\imath \omega, \imath \omega)=\tau+\Re e\left(\tau(\underline{\nu}+\bar{\nu}-2 \tilde{\varphi})+\frac{\theta}{\imath \omega}\right) \tilde{\kappa}_{\tilde{\varphi}}(\imath \omega)+\tau(\underline{\nu}-\tilde{\varphi})(\bar{\nu}-\tilde{\varphi})\left|\tilde{\kappa}_{\tilde{\varphi}}(\imath \omega)\right|^{2} .
$$

Here $\tilde{\kappa}_{\tilde{\varphi}}(\sigma)=c^{*}\left(\sigma I-A_{\tilde{\varphi}}\right)^{-1} b=\tilde{\kappa}(\sigma)(1+\tilde{\varphi} \tilde{\kappa}(\sigma))^{-1}$.

A straightforward computation shows that

$$
\tilde{\chi}_{\tilde{\varphi}}^{o}(-\imath \omega, \imath \omega)=\frac{1}{|1+\tilde{\varphi} \tilde{\kappa}(\imath \omega)|^{2}} \tilde{\chi}^{o}(-\imath \omega, \imath \omega)
$$

or

$$
\tilde{\chi}_{\tilde{\varphi}}^{o}(-\imath \omega, \imath \omega)=\left|\frac{\operatorname{det}(\imath \omega I-A)}{\operatorname{det}\left(\imath \omega I-A_{\tilde{\varphi}}\right)}\right|^{2} \tilde{\chi}^{o}(-\imath \omega, \imath \omega) .
$$

If $A$ has no eigenvalues on $\imath \mathbb{R}$ and $\tilde{\chi}^{o}(-\imath \omega, \imath \omega)>0$ for all $\omega>0$ including $\omega \rightarrow \infty$ then also $\tilde{\chi}_{\tilde{\varphi}}^{o}(-\imath \omega, \imath \omega)>0$ for all $\omega>0$ including $\omega \rightarrow \infty$. If $A$ has some 


\section{VLADIMIR RĂSVAN}

eigenvalues on $\imath \mathbb{R}$ and $\tilde{\chi}^{o}(-\imath \omega, \imath \omega) \geq 0$ only then (81) shows that $\tilde{\chi}_{\tilde{\varphi}}^{o}(-\imath \omega, \imath \omega) \geq 0$ also.

We assert now that (10) is exponentially stable for all linear functions $\phi(\nu)=$ $\tilde{\varphi} \nu$ where $\underline{\nu} \leq \tilde{\varphi} \leq \bar{\nu}$ or $\underline{\nu}<\tilde{\varphi}<\bar{\nu}$. This assertion is proved by contradiction: assume there exists some $\hat{\varphi} \in[\underline{\nu}, \bar{\nu}]$ or $\hat{\varphi} \in(\underline{\nu}, \bar{\nu})$ and some $\omega_{0} \in \mathbb{R}$ such that

$$
\begin{aligned}
\operatorname{det}\left(\imath \omega_{0} I-A+b \hat{\varphi} c^{*}\right) & =\operatorname{det}\left(\imath \omega_{0} I-A_{\tilde{\varphi}}+b(\hat{\varphi}-\tilde{\varphi}) c^{*}\right) \\
& =\left(\operatorname{det}\left(\imath \omega_{0} I-A_{\tilde{\varphi}}\right)\right)\left(1+(\hat{\varphi}-\tilde{\varphi}) \tilde{\kappa}_{\tilde{\varphi}}\left(\imath \omega_{0}\right)\right)=0 .
\end{aligned}
$$

Since $A_{\tilde{\varphi}}$ is a Hurwitz matrix we deduce $1+(\hat{\varphi}-\tilde{\varphi}) \tilde{\kappa}_{\tilde{\varphi}}\left(\imath \omega_{0}\right)=0$. If this equality is taken into account in (79) then

$$
\tilde{\chi}_{\tilde{\varphi}}^{o}\left(-\imath \omega_{0}, \imath \omega_{0}\right)=\tau \frac{(\underline{\nu}-\hat{\varphi})(\bar{\nu}-\hat{\varphi})}{(\tilde{\varphi}-\hat{\varphi})^{2}} \leq 0 .
$$

The equality is possible only for non-strict slope restrictions if $\tau>0$, but in this case the frequency domain inequality is strict for all $\omega$ including at $\infty$; the contradiction is obtained. In the critical cases, when the slope restrictions are strict and $\tau>0$, (82) is strict but this contradicts the non-strict frequency domain inequality of opposite type.

We have still to discuss some "limit" cases: $\tau=0$ and/or the frequency domain characteristic is constant for all $\omega$. In all these cases $\Im m \tilde{\kappa}(\imath \omega) \neq 0$ for all $\omega$ except $\omega=0$ hence $1+(\hat{\varphi}-\tilde{\varphi}) \tilde{\kappa}_{\tilde{\varphi}}\left(\imath \omega_{0}\right) \neq 0$ if $\omega_{0}=0$. But in this case we cannot have $1+(\hat{\varphi}-\tilde{\varphi}) \tilde{\kappa}_{\tilde{\varphi}}(0)=0$ because of the assumption $(1+\underline{\nu} \tilde{\kappa}(0))(1+\bar{\nu} \tilde{\kappa}(0))>0$.

In this way we obtained that (72) and (73) hold for all linear functions subject to the slope restrictions. Since the interval of the slope restrictions is compact, $\delta^{\varepsilon}$ and $\delta^{o}$ may be chosen independent of $\varphi$ to find

$$
\mathcal{W}_{\varphi}^{\varepsilon}(x) \geq \delta^{\varepsilon}|x|^{2}, \quad \mathcal{W}_{\varphi}^{o}(x) \geq \delta^{o}|x|^{2} .
$$

We can now proceed as follows: substitute in $\mathcal{W}_{\varphi}^{\varepsilon}$ or in $\mathcal{W}_{\varphi}^{o}$ the real number $\varphi$ by $\phi\left(c^{*} x\right) / c^{*} x$ for $c^{*} x \neq 0$ and by 0 if $c^{*} x=0$. It follows in both cases

$$
\begin{aligned}
\mathcal{W}(x) & =\left(A-b \phi\left(c^{*} x\right)\right)^{*} H\left(A-b \phi\left(c^{*} x\right)\right)+\frac{1}{2} \theta\left(c^{*} x\right) \phi\left(c^{*} x\right) \\
& =\mathcal{V}(x)+\theta\left(\int_{0}^{c^{*} x} \phi(\lambda) \mathrm{d} \lambda-\frac{1}{2}\left(c^{*} x\right) \phi\left(c^{*} x\right)\right) \geq \delta|x|^{2}
\end{aligned}
$$

which allows application of the following Liapunov like lemma of [10].

LEMma. Consider the system of ordinary differential equations in the general form

$$
\dot{x}=f(x), \quad \operatorname{dim} x=\operatorname{dim} f=n .
$$

Any constant vector $c$ satisfying $f(c)=0$ is called a stationary vector; the set of all stationary vectors is called stationary set. Assume there exists a continuous 


\title{
SLOPE RESTRICTED NONLINEARITIES
}

function $V: \mathbb{R}^{n} \mapsto \mathbb{R}$ with the following properties: i) $V(x(t))$ is nonincreasing with respect to $t$ along any solution of (85); ii) if for some bounded for $-\infty<t<\infty x(t), V(x(t)) \equiv$ const then this solution is a stationary vector; iii) $\lim _{|x| \rightarrow \infty} V(x)=\infty$. Then system (85) has global asymptotics, i.e., any of its solutions approaches for $t \rightarrow \infty$ the stationary set.

Combining now (84) with (41) and with the decrease conditions (68) and (170) we find that the assumptions of the Lemma hold for (10). Application of the Lemma to this system whose set of equilibria is the singleton $\{0\}$ proves Theorem 2 ,

\author{
Department of Automatic Control \\ University of Craiova \\ Department of Automatic Control \\ 13, A.I. Cuza str. \\ RO-200585-Craiova \\ ROMANIA \\ E-mail: vrasvan@automation.ucv.ro
}

